\title{
Unusual Case of Solitary Perineal Subcutaneous Metastasis From Sigmoid Colon Cancer
}

\author{
Taek-Gu Lee, Sang-Jeon Lee \\ Department of Surgery, Chungbuk National University College of Medicine, Cheongju, Korea
}

Subcutaneous metastasis from colorectal cancer is an unusual presentation. Most perineal subcutaneous metastases are found in extensive involvements of multiorgan metastases or local recurrences of rectal cancer. Subcutaneous metastasis from colon cancer is considered as a distant metastasis with poor prognosis. We report an unusual case of solitary subcutaneous metastasis beneath the perineum without solid organ involvement after a curative anterior resection for sigmoid colon cancer. The patient underwent a perineal resection, and chemotherapy with the FOLFOX (fluorouracil, leucovorin, and oxaliplatin) regimen was instituted. Eight months later, multiple lung metastases were found, and chemotherapy was restarted with the FOLFIRI (fluorouracil, leucovorin, and irinotecan) regimen. However, lung metastases progressed, and new metastases appeared at the adrenal glands, the kidneys and the cerebellum. The patient died 30 months after the diagnosis of perineal subcutaneous metastasis. He lived relatively long in comparison with patients in previous reports.

Keywords: Subcutaneous metastasis; Colorectal neoplasms

\section{INTRODUCTION}

Solitary subcutaneous metastasis from a visceral malignancy is rare. The incidences of cutaneous metastasis from a primary visceral tumor are from $0.7 \%$ to $9 \%$ [1-5], and those of cutaneous metastases from colorectal cancers are less than 4\% [1]. However, only one study on solitary subcutaneous metastasis from colorectal cancer has been reported because subcutaneous tissue as the first site of metastasis without other organ involvement is extremely rare. The most-commonly reported primary carcinomas resulting in clinically-recognized soft-tissue metastases are those of the lung, kidney, and colon $[1,2]$. The chest wall is the most common subcutaneous metastatic site. Pelvic recurrence is common in rectal cancer, but perineal recurrence after resection of colon cancer is considered to be a distant metastasis [6]. At the time of the diagnosis

Received: June 19, 2012 - Accepted: October 23, 2012

Correspondence to: Sang-Jeon Lee, M.D.

Department of Surgery, Chungbuk National University College of Medicine, 52 Naesudong-ro, Heungdeok-gu, Cheongju 361-804, Korea

Tel: +82-43-269-6360, Fax: +82-43-266-6037

E-mail:colon@chungbuk.ac.kr

(C) 2013 The Korean Society of Coloproctology

This is an open-access article distributed under the terms of the Creative Commons Attribution NonCommercial License (http://creativecommons.org/licenses/by-nc/3.0) which permits unrestricted noncommercial use, distribution, and reproduction in any medium, provided the original work is properly cited. of soft-tissue metastases, the metastasis is solitary in $17 \%$ of the cases, disseminated disease in $20 \%$, and concurrent with the primary cancer in 7\% [1-3]. It has a poor prognosis when it is considered to be a disseminated disease. The mean survival time for patients with soft-tissue metastasis has been reported to be 5.4 months [3]. Previous case reports are not detailed enough to provide any guidance for evaluation and treatment.

We report a rare case of a patient who presented with a solitary subcutaneous metastasis at the perineal area after a curative resection for sigmoid colon cancer. We hope that his clinical course can help provide optimal evaluation and treatment for a subcutaneous metastasis of colorectal cancer.

\section{CASE REPORT}

A 49-year-old man presented for a solitary perineal subcutaneous metastasis without other organ involvement. He had undergone an anterior resection with curative intent for sigmoid colon cancer located at $25 \mathrm{~cm}$ from the anal verge 10 months earlier. The histopathologic report revealed a moderately-differentiated adenocarcinoma infiltrating the proper muscle, with involvement of 7 out of 16 lymph nodes. There was neither lymphovascular invasion nor perineural invasion. The pathologic stage was T2N2bM0, stage IIIB according to American Joint Committee on Cancer 6th edition. Postoperative course was uneventful without any compli- 
Volume 29, Number 1, 2013

cations. Adjuvant chemotherapy with the FOLFOX (fluorouracil, leucovorin, and oxaliplatin) regimen was recommended. However, he refused the regimen and preferred an oral chemotherapeutic regimen with capecitabine. The patient received 24 weeks of treatment with oral capecitabine, $1,250 \mathrm{mg} / \mathrm{m}^{2}$ twice daily, days 1-14 every 21 days. Approximately 3 months after completion of the oral chemotherapy, elevation of the serum careinoembryonal antigen (CEA) level was found upon routine postoperative followup. He had no complaints, but a subcutaneous mass about $4 \mathrm{~cm}$ in diameter was palpated in the posterior perineal area on rectal examination with perineal palpation. It was rubbery hard, well demarcated and mildly tender. The inguinal lymph node was not palpated, and there was no palpable mass on the trunk. Serum tumor markers were elevated; CEA, $14.1 \mathrm{ng} / \mathrm{mL}$ and carbohydrate antigen 19-9, $157 \mathrm{U} / \mathrm{mL}$. Abdominopelvic computed tomography showed an ill-defined peripheral-enhancing low-attenuation mass, about $4.5 \mathrm{~cm}$ in maximal diameter, beneath the perineum (Fig. 1). The positron emission tomography-computed tomographic (PETCT) scan showed a hypermetabolic focus in perineal subcutaneous fat (SUVmax 8.6), suggesting that the mass was malignant (Fig. 2).

The patient underwent a perineal resection and transverse loop colostomy. Fig. 3 shows a solitary perineal subcutaneous metastasis from sigmoid colon cancer, which was confirmed as a metastatic adenocarcinoma upon histopathologic examination. Chemotherapy with the FOLFOX regimen was instituted. However, multiple lung metastases were detected on the PET-CT scan at 8 months after the perineal resection. The chemotherapy was switched to the FOLFIRI (fluorouracil, leucovorin, and irinotecan) regimen. The target agent was not affordable because of the patient's poor economic status. After the second cycle of FOLFIRI, some of the metastatic lesions had begun to progress, and new metastases had appeared at both the adrenal gland and the kidney. He visited the emergency department complaining of confused mental status, tremors and dyskinesia. A brain CT scan confirmed cerebellar metastasis. Whole brain radiotherapy was considered. The brain metastasis progressed rapidly, and he died 30 months after the diagnosis of perineal subcutaneous metastasis.

\section{DISCUSSION}

Subcutaneous metastasis from colorectal cancer is uncommon, subcutaneous metastasis; chest, trunk and extremities. Perineal subcutaneous metastasis from colon cancer is very rare. Most perineal or pelvic metastases are characterized by local recurrence of rectal cancer. Metastatic disease near the site of surgery is considered to be local, but perineal subcutaneous metastasis from colon cancer is considered to be remote. Lookingbill et al. [5] showed that the incidence of cutaneous metastasis was 420 of 4,020 meta-

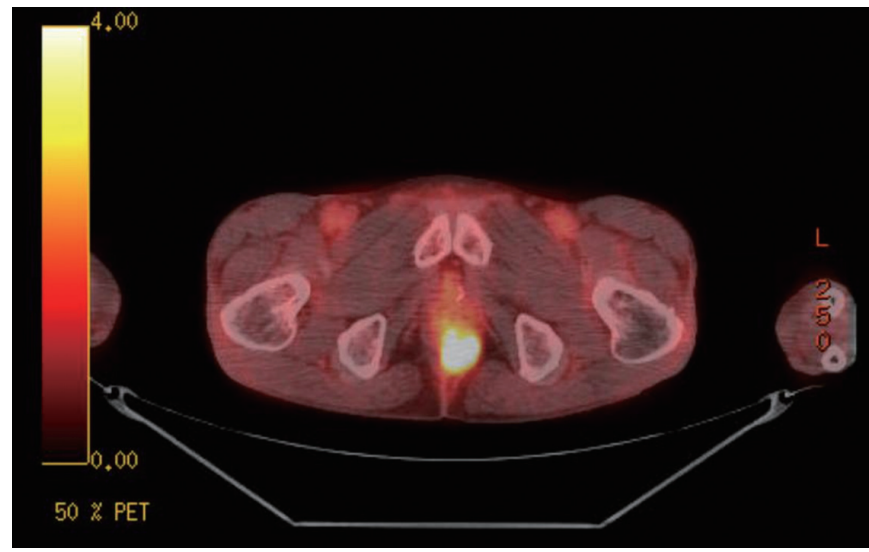

Fig. 2. Positron emission tomography-computed tomographic scan showing a hypermetabolic focus in subcutaneous fat (SUVmax 8.6).
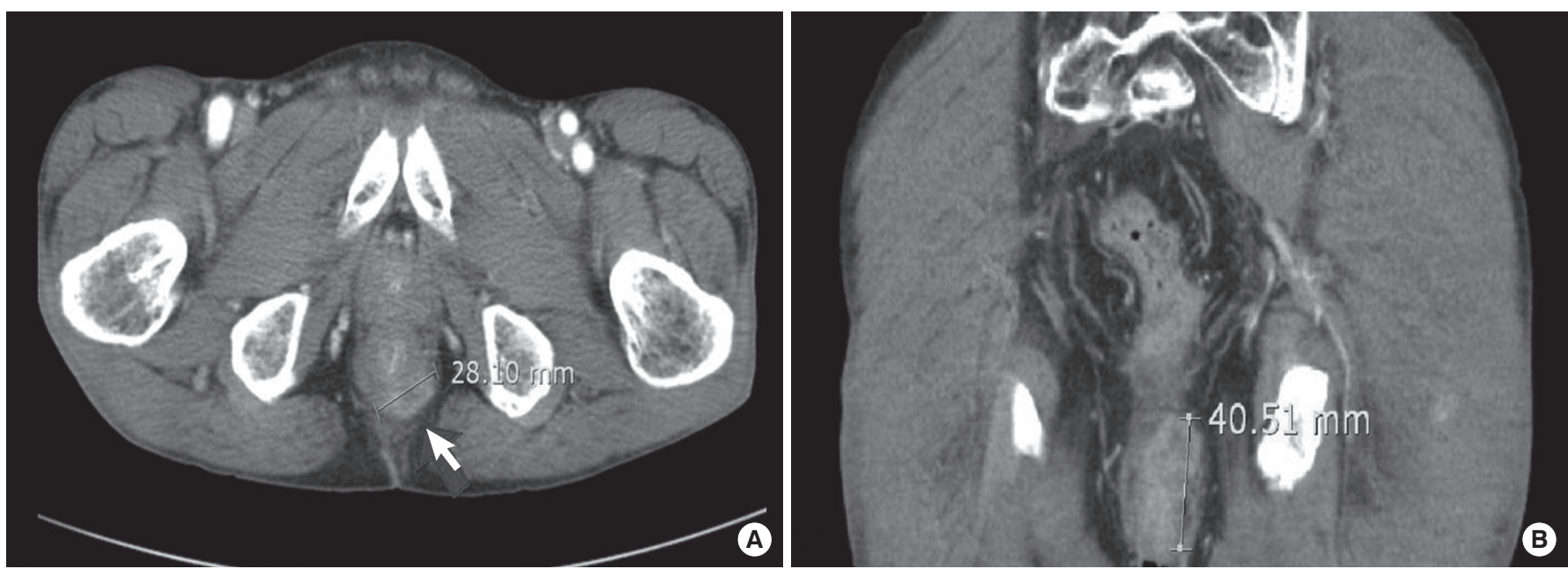

Fig. 1. Abdominal computed tomographic scan of a 49-year-old man: (A) axial section showing a subcutaneous mass beneath the perineum and (B) coronal view showing a large subcutaneous mass measuring $4.1 \mathrm{~cm} \times 2.8 \mathrm{~cm}$. 


\section{Coloproctology Taek-Gu Lee and Sang-Jeon Lee}
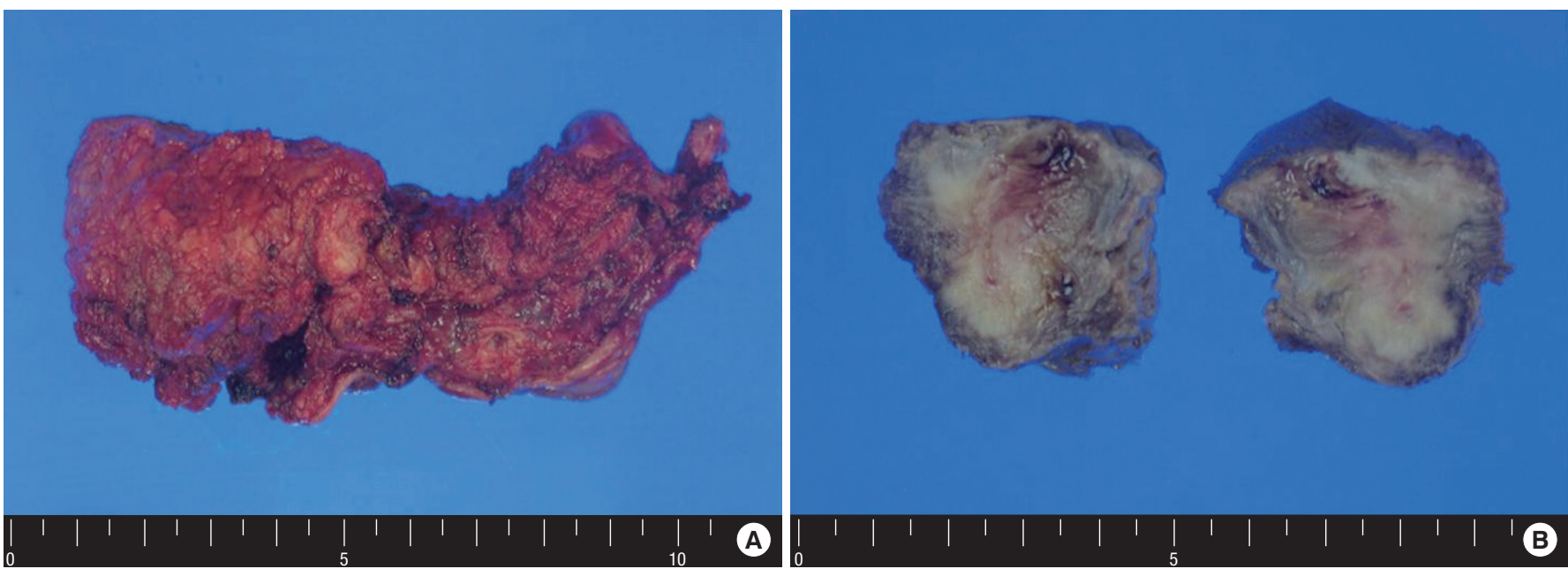

Fig. 3. Surgical specimen: (A) fresh specimen and (B) subcutaneous mass fixed with formalin.

static carcinoma registry patients. Among cutaneous metastases from 18 colorectal cancer patients, remote metastases were observed in 7 patients (38.9\%). Eleven of the 18 patients with colorectal cancer had only local metastases located in the abdominal incision site. In remote metastasis, the metastatic nodule is most commonly located on the trunk, not in the pelvis.

The overall incidence of cutaneous metastasis from a visceral neoplasm is $5.3 \%$, ranging from $0.7 \%$ to $9 \%$ [1-5]. Breast cancer has the highest incidence of cutaneous metastasis. Lung cancer, colorectal cancer, renal cancer, ovarian cancer, and bladder cancer all have similar rates of cutaneous metastasis of between $3.4 \%$ and $4 \%$. The incidence of cutaneous metastasis was $5 \%$ for 7,316 tumor registry patients, and among these, $1.3 \%$ of the patients had a cutaneous metastasis at the time of diagnosis [1]. The incidence of cutaneous metastasis from colorectal cancer ranges from $2.3 \%$ to $3.4 \%$, and the incidence of metastasis in the pelvis is $6 \%$. Damron and Heiner [2] in a retrospective study of 91 cases found that a minority of the cases had widespread metastases. The most common site for soft-tissue metastasis is the thigh muscle, and the most common cancer is lung cancer. The most common histological diagnosis overall is an adenocarcinoma, most frequently from the lung or gastrointestinal tract. However, reports of a solitary subcutaneous metastasis are mostly case reports because a subcutaneous metastasis without other organ involvement is extremely rare $[6,7]$.

The symptoms of a metastatic carcinoma to soft tissue are similar to those of a soft-tissue sarcoma in many respects, but a painful soft-tissue mass is more commonly noted in patients with softtissue metastasis than in patients with a primary sarcoma. Softtissue metastases, especially those in skeletal muscles, are frequently painful. Cutaneous involvement of colorectal cancer at the time of diagnosis appeared as an inflammatory abscess of the perineum and a palpable nodule in a recent study [5]. However, this patient had no symptoms because the metastatic tumor was limited to the subcutaneous tissue in this case; the mass was detected on physical examination during routine postoperative surveillance for sigmoid colon cancer.

In clinical practice, when skin metastases appear, they are usually associated with extensive disseminated disease, rapid tumor progression, and poor prognosis secondary to widespread visceral involvement. Patients with a Sister Mary Joseph `s nodule in a gastric carcinoma, a metastatic nodule to the umbilicus that most commonly is a rare phenomenon, portends a poor prognosis [8].

The mean survival time of patients with cutaneous metastasis ranges from 1 to 34 months, depending on the primary tumor [5]. Patients with skin metastasis from colorectal cancer have an average survival of 18 months. Schoenlaub et al. [9] also reported similar results. They noted that the median survival time of patients with cutaneous metastasis from a visceral primary tumor was 6.5 months, and especially, the median survival time of patients with a colorectal primary tumor was even worse, being 4.4 month. Bordin and Weitzner [10] reported that the duration of survival from the time of diagnosis of the metastatic carcinoma in the skin averaged 11.4 weeks, with a range of 2 to 34 weeks. The interval between the diagnosis of the primary malignancy and the subcutaneous metastases varied between one and two years.

The patient in our case received a perineal resection for subcutaneous metastasis and chemotherapy with the FOLFOX regimen. Eight months later, he was readmitted because of multiple lung metastases and restarted chemotherapy with the FOLFIRI regimen. He died 30 months after the diagnosis of perineal subcutaneous metastasis. He lived relatively long in comparison with previous reports. This suggests that proper surgical resection and systemic chemotherapy help to prolong survival even though patients with subcutaneous metastasis typically have widespread dissemination of the disease and a poor prognosis. 


\section{CONFLICT OF INTEREST}

No potential conflict of interest relevant to this article was reported.

\section{ACKNOWLEDGMENTS}

This work was supported by the research grant of the Chungbuk National University in 2011.

\section{REFERENCES}

1. Lookingbill DP, Spangler N, Sexton FM. Skin involvement as the presenting sign of internal carcinoma: a retrospective study of 7316 cancer patients. J Am Acad Dermatol 1990;22:19-26.

2. Damron TA, Heiner J. Distant soft tissue metastases: a series of 30 new patients and 91 cases from the literature. Ann Surg Oncol 2000;7:526-34.

3. Plaza JA, Perez-Montiel D, Mayerson J, Morrison C, Suster S. Metastases to soft tissue: a review of 118 cases over a 30-year period. Cancer 2008;112:193-203.
4. Krathen RA, Orengo IF, Rosen T. Cutaneous metastasis: a metaanalysis of data. South Med J 2003;96:164-7.

5. Lookingbill DP, Spangler N, Helm KF. Cutaneous metastases in patients with metastatic carcinoma: a retrospective study of 4020 patients. J Am Acad Dermatol 1993;29(2 Pt 1):228-36.

6. Herrera-Ornelas L, Petrelli N, Mittelman A. Subcutaneous metastases without visceral metastases from an adenocarcinoma of the rectum. Dis Colon Rectum 1985;28:523-5.

7. Tan KY, Ho KS, Lai JH, Lim JF, Ooi BS, Tang CL, et al. Cutaneous and subcutaneous metastases of adenocarcinoma of the colon and rectum. Ann Acad Med Singapore 2006;35:585-7.

8. Schwartz IS. Sister (Mary?) Joseph's nodule. N Engl J Med 1987; 316:1348-9.

9. Schoenlaub P, Sarraux A, Grosshans E, Heid E, Cribier B. Survival after cutaneous metastasis: a study of 200 cases. Ann Dermatol Venereol 2001;128:1310-5.

10. Bordin GM, Weitzner S. Cutaneous metastases as a manifestation of internal carcinoma: diagnostic and prognostic significance. Am Surg 1972;38:629-34. 